\title{
THE USE OF TORQUE GENERATORS TO STUDY CLONUS AND DISTURBANCES OF MUSCLE TONE
}

\author{
By P. HARRIS and E. G. WALSH \\ Departments of Neurological Surgery and Physiology, University of Edinburgh
}

UNTIL recently the neurophysiological investigation of normal and pathological motor control systems has been handicapped by the lack of a suitably versatile tool to enable forces to be applied to the limb under consideration. This gap has now been closed since devices known as torque generators became available. It is possible by supplying appropriately controlled electric currents to produce the corresponding mechanical transform with the limb coupled to the device. For clinical purposes the most suitable of the generators is the printed motor; this is the same device which is used to move tape in computers. In the past 3 years one of us has been concerned with the reactions of Parkinsonism patients (Walsh, 1969, 1970), but recently, studies have included some 6 paraplegic patients. For the study of the knee or ankle, the largest of the standard range of these motors (type Gi6 M4) is satisfactory. The motor can be supplied with currents which alternate at predetermined rates whilst the motion of the limb is registered by a high grade potentiometer. We are currently using this system to study the effects of drug therapy (Diazepam; (Valium-Roche Products Ltd.)), and of operative procedures such as neurectomy and anterior rhizotomy. In some of these patients, the knee appears to be sharply resonant when excited at about $2 \mathrm{~Hz}$ (two cycles per second). These figures obtained with the axis of the knee vertical, indicate that at this rate a mechanical force produces substantially more movement than when the rate is higher or lower. For the study of clonus also, the method is very useful, producing quite unexpected results as to the mechanisms underlying the rhythmicity (Walsh, I97I). Our methods clearly need further development, but they do appear to be of value as an objective method for the assessment of certain motor disturbances.

During this study some other observations were made, including an interesting reflex effect in a paraplegic patient, and this is described in the following paper.

\section{REFERENCES}

Walsh, E. G. (1969). F. Physiol. 202, I09.

Walsh, E. G. (1970). F. Physiol. 209, 33.

WALsh, E. G. (I971). F. Physiol. (in press). 